\title{
Análisis de sobrevida del cáncer gástrico en anillo de sello según profundidad y compromiso linfonodal ${ }^{*}$ Survival of patients with signet ring cell carcinoma of the stomach, compared with patients with non ring cell gastric cáncer
}

\author{
Drs. FRANCISCO VENTURELLI M.1,2, CARLOS CÁRCAMO I.1,2, ALIRO VENTURELLI L. 1,2, \\ MARCELA CÁRCAMO B. ${ }^{4}$, MARIANNE BORN G. ${ }^{1,2}$, CRISTIÁN JARA D. ${ }^{1,2}$, \\ EDGARDO MANSILLA S. ${ }^{1,2}$, CRISTIAN CARRASCO L. ${ }^{3}$, ORLANDO FELMER E $E^{1,2}$
}

${ }^{1}$ Instituto de Cirugía. Universidad Austral, ${ }^{2}$ Servicio de Cirugía. Hospital Clínico Regional Valdivia, ${ }^{3}$ Servicio de Anatomía Patológica. Hospital Clínico Regional Valdivia, ${ }^{4}$ Departamento de Salud Pública y Epidemiología, Universidad de los Andes, Plataforma Investigación Instituto Nacional del Cáncer, Santiago, Chile.

\section{RESUMEN}

Objetivo: Comparar la sobrevida del cáncer gástrico en anillo de sello (AS) con los otros tipos de adenocarcinoma (no anillo de sello (NAS)), según el compromiso en profundidad de la pared gástrica (T) y los números de linfonodos comprometidos (N). Material y Métodos: Se realizó un estudio de cohorte retrospectivo, en el cuál se seleccionó a todos los pacientes gastrectomizados por adenocarcinoma gástrico en el Hospital Base Valdivia entre los años 1988-2005. Se compararon los pacientes cuyo informe histopatológico concluía que el cáncer gástrico era un AS (más del $50 \%$ de sus células son anillo de sello) con los que no lo eran (NAS). En el análisis estadístico se utiliza el test de $\chi^{2}$, Método de Kaplan Meier, test de Log Rank. Resultados: Total 287 casos. 60 AS y 227 NAS. No hubo diferencias estadísticamente significativas en cuanto a la profundidad tumoral entre ambos grupos. Los AS presentaron mayor compromiso linfonodal. Al comparar la sobrevida a 5 años de los AS con los NAS por estadio (TNM), no encontramos diferencias significativas. Conclusión: A la luz de este trabajo el tipo histológico anillo de sello no parece constituir un factor de mal pronóstico en el cáncer gástrico.

\section{PALABRAS CLAVE: Pronóstico, cáncer, anillo de sello, estudio de cohorte.}

\begin{abstract}
Background: Signet ring cell (SRC) carcinoma of the stomach corresponds to 3 to $39 \%$ of all cases of gastric cancer. It tends to be less differentiated, infiltrative and diffuse. It is not clear if it has a different prognosis than other types of gastric cancer. Aim: to compare the survival of SRC gastric carcinoma with other types of gastric cancer, considering depth of gastric wall involvement and number of infiltrated lymph
\end{abstract}

\footnotetext{
* Recibido el 18 de Diciembre de 2007 y aceptado para publicación el 24 de Marzo de 2008.

Correspondencia: Dr. Francisco J. Venturelli M.

Los Manzanos 365. Isla Teja, Valdivia, Chile

E-mail: icoventurelli@gmail.com
} 
nodes. Material and Methods: Retrospective review of pathology samples of all gastric cancers operated between 1988 and 2005. Survival of patients with and without SRC gastric cancer was compared. Results: A total of 287 cases were reviewed. Of these 60 had a SRC gastric cancer. No differences in gastric wall infiltration between SRC and non SRC gastric cancer was observed. SRC gastric cancer has a higher frequency of lymph node involvement. No differences in survival between SRC and non SRC gastric cancer, were observed. Conclusions: In this retrospective series of patients, no differences in survival were observed between SRC and non SRC gastric cancer.

\section{KEY WORDS: Signet ring cell carcinoma, gastric cancer, survival.}

\section{INTRODUCCIÓN}

En Chile, el Cáncer Gástrico es la principal causa de muerte por cáncer con una tasa de mortalidad de 20/100.000 habitantes ${ }^{1}$ y se presenta más frecuentemente en el sexo masculino $(63 \%)^{2}$. Sus factores pronósticos están dados principalmente por el compromiso linfonodal y el grado de invasión de la pared gástrica ${ }^{3,4}$.

Aunque diversos autores muestran que este tumor se localiza preferentemente en el antro del estómago ${ }^{3,5,6}$, en los últimos años, se ha visto que la localización ha ido cambiando al tercio superior $^{1,6}$. Así también ha ido cambiando el tipo histológico de un patrón diferenciado a un mal diferenciado ${ }^{5}$.

El cáncer gástrico de AS se origina en la mucosa gástrica a nivel del cuello glandular de la zona proliferativa del estómago ${ }^{7}$ y se caracteriza por presentar una disminución de la expresión de las proteínas E caderinas, lo cual está asociado a un fenotipo infiltrativo y a un peor pronóstico ${ }^{8}$. De acuerdo a la clasificación de la World Health Organization (WHO) ${ }^{9}$ el cáncer AS es aquel que presenta más del $50 \%$ de células en anillo de sello en el estudio histopatológico del estómago resecado.

Los AS corresponden al 3-39\% ${ }^{10,11}$, de los cánceres gástricos y se caracterizan por ser mal diferenciados, infiltrativos y difusos, y por comprometer más frecuentemente toda la pared del estómago y presentar un mayor compromiso linfonodal ${ }^{10-12}$.

Se han publicado varios trabajos que analizan la sobrevida de este tipo histológico en el estómago, obteniéndose resultados contradictorios. Algunos, han demostrado un mejor pronóstico ${ }^{13,14}$, otros un peor pronóstico ${ }^{15} \mathrm{y}$ otros no demuestran diferencias estadísticamente significativas, al compararlo con los de tipo histológico no anillo de sello (NAS) $^{10,11}$. Aquellos trabajos que muestran que el AS presenta un peor pronóstico, asocian como factor influyente un mayor compromiso linfonodal, concluyendo, que el tipo histológico no es un factor pronóstico en esta patología ${ }^{11}$.

El objetivo de este estudio es comparar la sobrevida del cáncer gástrico AS con los NAS se- gún el compromiso en profundidad de la pared gástrica (T) y el número de linfonodos comprometidos $(\mathrm{N})$.

\section{MATERIAL Y MÉTODO}

Se realizó un estudio de cohorte retrospectivo, en el cual se selecciona a todos los pacientes (423) gastrectomizados por adenocarcinoma gástrico en el Hospital Base Valdivia entre enero de 1988 y diciembre del 2005. Un solo patólogo, revisó los informes anatomopatológicos realizados por su equipo de trabajo, utilizando los criterios de la WHO para definir el tipo histológico. Se excluyeron en total 136 pacientes; 50 por presentar un informe histopatológico incompleto o insuficiente, definidos como aquellos que no presentaban datos en las variables estudiadas (missing), 51 casos por no se obtener antecedentes en el registro civil de su fecha de muerte y 35 casos por tener una linfadenectomía insuficiente para ser clasificados según la TNM (< a 15 linfonodos).

De los pacientes resultantes (287), se seleccionaron aquellos con diagnóstico de adenocarcinoma AS y se comparó su sobrevida con el resto de los casos (NAS).

Los grupos se compararon usando el test de $\chi^{2}$. La proporción de supervivencia a 5 años y mediana de supervivencia se estimó estratificando siempre según fueran AS o NAS, considerando la clasificación TNM. Para la descripción de las curvas de sobrevida, se utilizó el método de Kaplan Meier, determinando como fecha de inicio de seguimiento en el momento de la cirugía gástrica.

Las curvas de supervivencia fueron comparadas utilizando los test de Log Rank con un intervalo de confianza de un 95\%. La información se procesa utilizando el programa Statistical Package for the Social Sciences, (SPSS, versión 12).

\section{RESULTADOS}

Del total de casos analizados 60 pacientes $(20,9 \%)$ se clasificaron como AS y $227(79,09 \%)$ 
como NAS. El promedio de edad para los anillo de sello fue menor que para los no anillo de sello $(p=0,0000)$. En cuanto al sexo no hubo diferencias significativas $(p>0,05)$, pero si una tendencia a que en los anillo de sello, hubo proporcionalmente más mujeres (Tabla 1).

Del total de pacientes 27 casos $(9,4 \%)$ fueron incipientes y 260 (90,59\%) avanzados. De los 27 casos de cáncer incipiente sólo 6 (22\%) fueron AS.

No hubo diferencias estadísticamente significativas en cuanto a la profundidad tumoral entre ambos grupos, pero donde si la hubo fue en el compromiso linfonodal, donde los AS presentaron un mayor compromiso $(p=0,003)$ (Tabla 2). En cuanto a el estadio al momento del diagnóstico, los anillos de sello se presentaron en un estadio más avanzado de la enfermedad $(p=0,009)$ (Tabla 3$)$.

\section{Supervivencia}

Tanto en el estadio IA, como en el IB y como en el IIIB, la sobrevida a 5 años fue más alta en los anillo de sello, pero no se alcanzó significancia estadística (EI IA: $63,98 \%$ para los NAS y de $100 \%$ para los anillo de sello $(p=0,155)$ (Figura 1).

El IB: $57,72 \%$ para los NAS y de $100 \%$ para los anillo de sello $(p=0,260)$ (Figura 2$)$.

El IIIB: $22,63 \%$ para los NAS y de $23,21 \%$ para los anillo de sello ( $p=0,352)$ (Figura 5 ).

Tabla 1

\section{CARACTERÍSTICAS DEMOGRÁFICAS}

\begin{tabular}{|c|c|c|c|c|c|}
\hline \multirow[b]{2}{*}{ Edad } & \multicolumn{2}{|c|}{$\begin{array}{c}\text { No Anillo de Sello } \\
\quad n=227\end{array}$} & \multicolumn{2}{|c|}{$\begin{array}{c}\text { Anillo de Sello } \\
\quad n=60\end{array}$} & \multirow{2}{*}{$\begin{array}{c}p \text { valor } \\
p=0,0000\end{array}$} \\
\hline & 65 & $(20-86)$ & 54 & $(23-81)$ & \\
\hline Sexo & & & & & $p=0,756$ \\
\hline Masc & 149 & $(65,63 \%)$ & 38 & $(63,33 \%)$ & \\
\hline Fem & 78 & $(34,36 \%)$ & 22 & $(36,66 \%)$ & \\
\hline Total & 227 & $(100 \%)$ & 60 & $(100 \%)$ & \\
\hline
\end{tabular}

Tabla 2

CARACTERÍSTICAS CLÍNICO PATOLÓGICAS

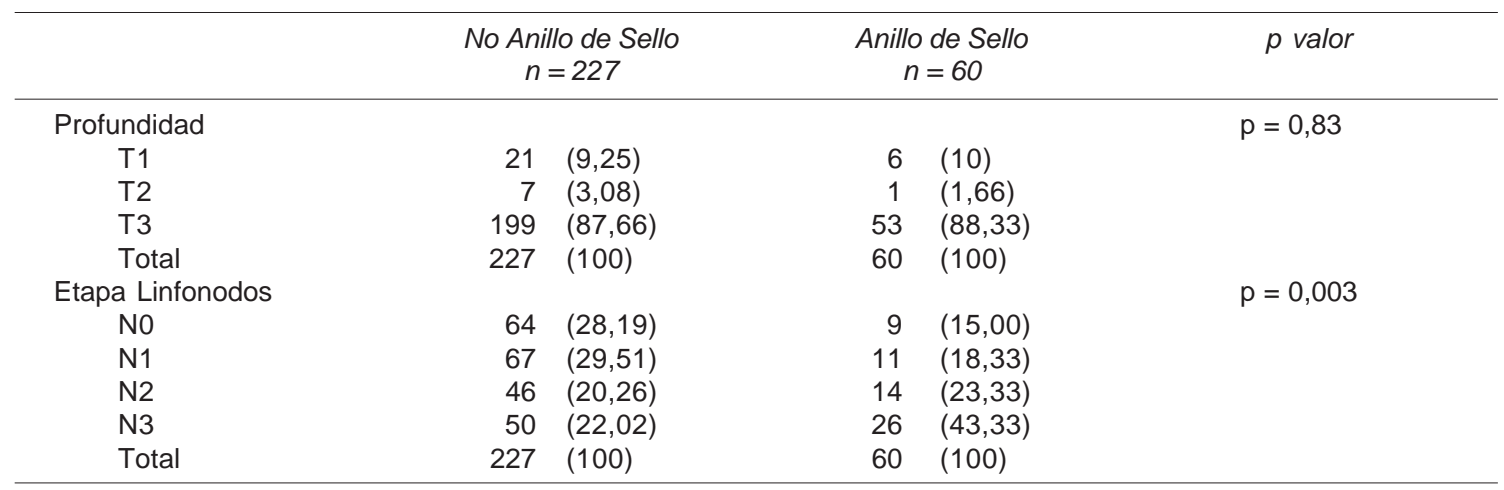

Tabla 3

CARACTERÍSTICAS CLÍNICO PATOLÓGICAS

\begin{tabular}{|c|c|c|c|c|c|}
\hline & \multicolumn{2}{|c|}{ No Anillo de Sello } & \multicolumn{2}{|c|}{ Anillo de Sello } & \multirow[t]{2}{*}{$p$ valor } \\
\hline & & & & (\%) & \\
\hline \multicolumn{6}{|l|}{ Estadio } \\
\hline $\mathrm{IA}$ & 16 & $(7,05)$ & 4 & $(6,67)$ & \\
\hline IB & 11 & $(4,85)$ & 3 & $(5,00)$ & \\
\hline II & 43 & $(18,34)$ & 4 & $(6,67)$ & $p=0,009$ \\
\hline IIIA & 61 & $(26,87)$ & 9 & $(15,00)$ & \\
\hline IIIB & 46 & $(20,26)$ & 14 & $(23,33)$ & \\
\hline IV & 50 & $(22,03)$ & 14 & $(43,33)$ & \\
\hline Total & 227 & $(100)$ & 60 & $(100)$ & \\
\hline
\end{tabular}


Análisis de sobrevida del cáncer gástrico en anillo de sello según profundidad y ... / Francisco Venturelli M. y cols.

En el estadio II, IIIA y IV, la sobrevida a 5 años fue mayor en los no anillo de sello, pero tampoco alcanzó significancia estadística (El II: 68,73\% para los NAS y $33,33 \%$ para los anillo de sello $(p=0,298)$ (Figura 3).

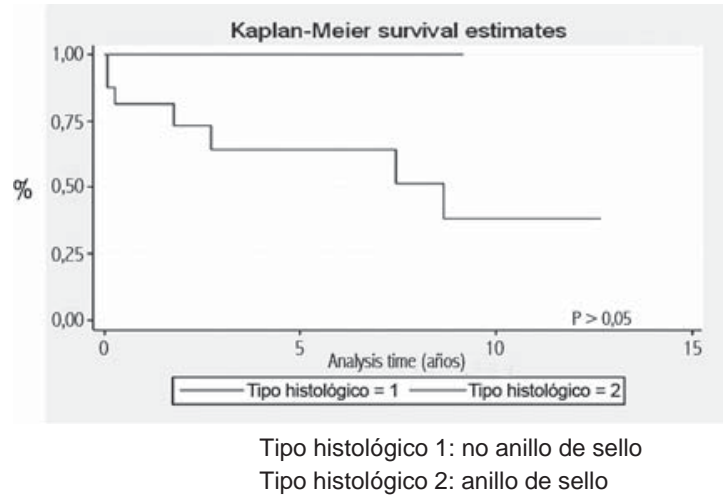

Figura 1. Curva de sobrevida de pacientes con cáncer gástrico estadio IA según tipo histológico.

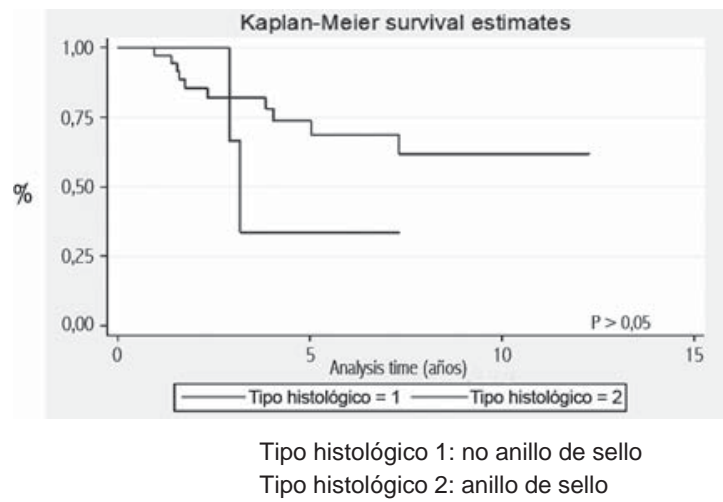

Figura 3. Curva de sobrevida de pacientes con cáncer gástrico II según tipo histológico.

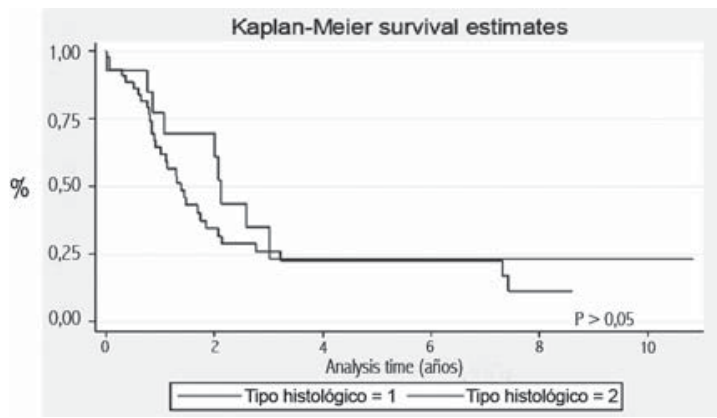

Tipo histológico 1: no anillo de sello Tipo histológico 2: anillo de sello

Figura 5. Curva de sobrevida de pacientes con cáncer gástrico IIIB según tipo histológico.
El IIIA: $33,34 \%$ para los NAS y de $12,5 \%$ para los anillo de sello $(p=0,053)$ (Figura 4$)$.

El IV $13,13 \%$ para los NAS y de $0,53 \%$ para los anillo de sello $(p=0,264)$ (Figura 6$)$.

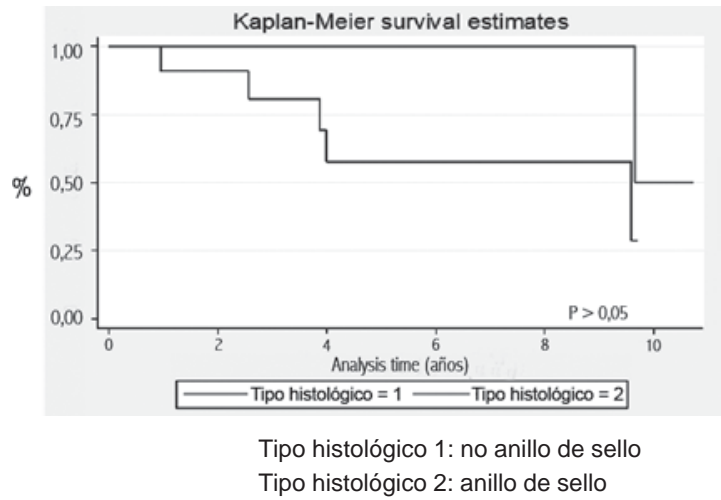

Figura 2. Curva de sobrevida de pacientes con cáncer gástrico IB según tipo histológico.

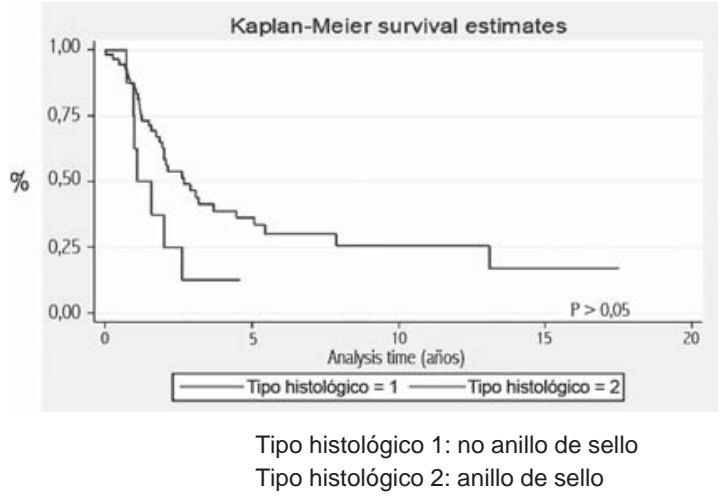

Figura 4. Curva de sobrevida de pacientes con cáncer gástrico IIIA con compromiso linfático según tipo histológico.

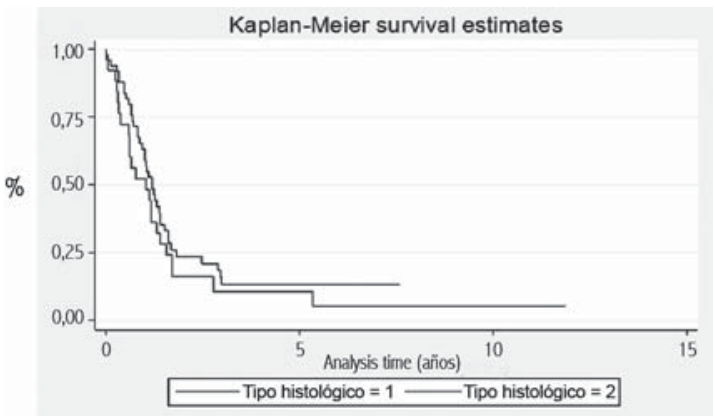

Tipo histológico 1: no anillo de sello Tipo histológico 2: anillo de sello

Figura 6. Curva de sobrevida de pacientes con cáncer gástrico IV según tipo histológico. 


\section{DISCUSIÓN}

Según algunos autores el tipo histológico AS ensombrece el pronóstico de los pacientes que padecen de cáncer gástrico ${ }^{10,15}$; sin embargo, cuando este tumor no sobrepasa la submucosa (cáncer gástrico incipiente), se ha visto que la sobrevida a 5 años es mayor que en los NAS ${ }^{11,13,14}$. Está en discusión si la peor sobrevida de los pacientes con AS avanzados (comprometen más allá de la submucosa) es por el compromiso linfonodal y/o por la profundidad que presentan al momento del diagnóstico, o si es porque este tipo histológico es un factor independiente en la sobrevida.

Los AS representan entre el $3-39 \%$ de los cánceres gástricos $7,10,11,14$. En nuestro estudio la prevalencia fue de un $20,90 \%$, lo que corresponde a lo publicado internacionalmente.

El compromiso linfonodal es el principal factor pronóstico en el cáncer gástrico ${ }^{3}$. Muchos cirujanos creen que los AS presentan un peor pronósti$\mathrm{co}$, porque al momento del diagnóstico estos tendrían un mayor compromiso linfonodal que los NAS; sin embargo, hay estudios recientes que demuestran similar compromiso linfonodal para ambos gru$\operatorname{pos}^{14,16}$. En nuestro estudio, los AS al momento del diagnóstico tuvieron un mayor compromiso linfonodal en forma estadísticamente significativa al compararlos con los NAS, lo cual significó a su vez que sean clasificados dentro de estadios más avanzados.

Eigo Otsuji et al ${ }^{15}$, así como Dong Yi Kim et al ${ }^{11}$, reportaron que la sobrevida a 5 años del tipo histológico AS era mejor que en los NAS, pero Eigo Otsuji et $\mathrm{al}^{15}$, al separar los avanzados de los incipientes vio que los incipientes AS presentaban una mejor sobrevida, y que en los avanzados no había una diferencia significativa al comparar AS vs NAS. En nuestro estudio al comparar la sobrevida a 5 años de los AS vs no AS por estadio no encontramos diferencias estadísticamente significativas, lo cual es concordante con el trabajo de Theuer CP et al ${ }^{10}$.

\section{CONCLUSIÓN}

Los AS presentan un mayor compromiso linfonodal que los NAS. El tipo histológico anillo de sello no parece constituir un factor de mal pronóstico en el cáncer gástrico.

\section{REFERENCIAS}

1. Csendes A, Burdiles P, Braghetto I, Díaz J, Maluenda F, Korn O, y cols. Resecabilidad y mortalidad operatoria de la gastrectomía subtotal y total en pacientes con cáncer gástrico avanzado, entre 1969 y 2004. Rev Méd Chile 2006; 134: 426-432.
2. Jarufe N, Covacevich S, Burmeister R, Rubilar P, García C. Cáncer gástrico en menores de 40 años Rev Chil Cir 1999; 51: 395-400.

3. Yokota T, Ishiyama S, Saito T, Teshima S, Narushima $\mathrm{Y}$, Murata $\mathrm{K}$, et al. Lymph node metastasis as a significant prognostic factor in gastric cancer: a multiple logistic regression analysis. Scand J Gastroenterol 2004; 39: 380-384.

4. Venturelli A, Cardemil B, Díaz J, Avendaño R, Murúa $A$, Kuschel $C$, y cols. Cirugía más quimioterapia en cáncer gástrico: sobrevida a 5 años. Rev Chil Cir 1999; 51: 170-175.

5. Earl D, Dittus Ch, Younes M, Nguyen H, AlboresSaavedra J. Differential Trends in the Intestinal and Diffuse Types of Gastric Carcinoma in the United States 1973-2000 Increase in the Signet Ring Cell Type. Arch Pathol Lab Med 2004; 128: 765-770.

6. Popiela T, Kulig J, Ko Odziejczyk P, Sierzega M. Changing patterns of gastric carcinoma over the past two decades in a single institution: clinicopathological findings in 1557 patients. Scand J Gastroenterol 2002; 37: 561-567.

7. Takashi Yokota, Yasuo Kunii. Signet ring cell carcinoma of the stomach: A Clinicopathological Comparison with the Others Histological Types. Tohoku J Exp Med 1998; 186; 121-130.

8. Charlton A, Blair V, Shaw D, Parry S, Guilford P, Martin IG. Hereditary diffuse gastric cancer: predominance of multiple foci of signet ring cell carcinoma in distal stomach and transitional zone. Gut 2004; 53: 814-820.

9. Hamilton S, Aaltonen L. Tumors of the Stomach. En: Pathology and Genetics of Tumours of the Digestive System. Lyon, France: IARC Press; 2000; 44.

10. Theuer CP, Nastanski F, Brewster WR, Butler JA, Anton-Culver $\mathrm{H}$. Signet ring cell histology is associated with unique clinical features but does not affect gastric cancer survival. Am Surg 1999; 65: 915-921.

11. Kim DY, Park YK, Joo JK, Ryu SY, Kim YJ, Kim SK, et al. Clinicopathological characteristics of signet ring cell carcinoma of the stomach. ANZ J Surg 2004; 74: 1060-1064.

12. Kim DY Clinicopathological characteristics of gastric carcinoma in young patients. Langenbecks Arch Surg 2003; 388: 245-249.

13. Hyung WJ, Noh SH, Lee JH, Huh JJ, Lah KH, Choi $\mathrm{SH}$, et al. Early gastric carcinoma with signet ring cell histology. Cancer 2002; 94: 78-83.

14. Kunisaki C, Shimada H, Nomura M, Matsuda G, Otsuka Y, Akiyama H. Therapeutic strategy for signet ring cell carcinoma of the stomach. Br J Surg 2004; 91: 1319-1324.

15. Otsuji E, Yamaguchi T, Sawai K, Takahashi T. Characterization of signet ring cell carcinoma of the stomach. J Surg Oncol 1998; 67: 216-220.

16. Dong Yi Kim, Kyeung Won Seo, Jae Kyoon Joo, Young Kyu Park, Seong Yeob Ryu, Hyeong Rok Kim, et al. Prognostic factors in patients with nodenegative gastric carcinoma: A comparison with node-positive gastric carcinoma. World J Gastroenterol 2006; 12: 1182-1186. 